\title{
11. DISCUSSION FOLLOWING THE REPORTS BY PARKER AND VERSCHUUR
}

(Friday, September 12, 1969)

Chairman: S. B. PIKEL'NER

Editor's remarks: The Discussion has been rearranged into four sections: 1. Various Questions; 2. The Galactic Halo; 3. The Origin of the Magnetic Field; 4. Summary. Section 4 is the outcome of another meeting (on September 17). One very extensive remark has been condensed. The latter part of the discussion on September 12 dealt with the informal meeting on $\mathrm{H} I$ gas and is presented separately as Chapter 6.

\section{Various Questions}

Van Woerden: Parker uses a value of $0.2 \mathrm{~cm}^{-3} \mathrm{kpc}$ for the product $n_{\mathrm{H}} \Lambda$. This product is equivalent to the integral $\int_{0}^{\infty} n_{\mathrm{H}} \mathrm{d} z$. I estimate $0.1 \mathrm{~cm}^{-3} \mathrm{kpc}$ as an average over the ring between 9 and $11 \mathrm{kpc}$. The estimate comes from unpublished work, based on integrations in the diagrams published by Westerhout (1957). This estimate may be too low because of molecular hydrogen and because of concentrations of atomic hydrogen in dense clouds. (Westerhout, G.: 1957, Bull. Astron. Inst. Netherl. 13, 201.)

Burke: A model-independent value for the integral can be derived for the solar neighborhood from observations in the direction of the pole. What is this number averaged over all latitudes above, say, $40^{\circ}$ ?

Van Woerden: The high-latitude survey by Tolbert (1970) gives a value close to $0.1 \mathrm{~cm}^{-3} \mathrm{kpc}$. (Tolbert, C. R.: 1970, in preparation.)

Field: I am surprised that neither of our speakers discussed the strong magnetic field (i.e., a field of $10 \mu \mathrm{G}$ or more) which has been the subject of controversy at many previous symposia. Evidence for a strong field was derived from the intensity of synchrotron radiation, together with estimates of the flux of cosmic-ray electrons near the Earth. A recent study by Anand et al. (1969) concludes that at present such arguments yield $6 \mu \mathrm{G}$ as the best value. The difference is due to the postulate of a very significant solar modulation of electrons below about $1 \mathrm{GeV}$. This means that the number of radiating electrons is considerably larger in interstellar space than close to the Earth. Consequently, the magnetic field required to explain the synchrotron emission is lower than the previous estimate. It is interesting that the value of $6 \mu \mathrm{G}$ is comparable to the numbers which were derived from other lines of evidence and were mentioned by Verschuur in his report. (Anand, K. C., Daniel, R. R., and Stephens, S. A.: 1968, Proc. Ind. Acad. Sci. 67, 267.)

Davis: Is the information on modulation sufficiently certain so that in another two years the field may not have dropped a bit more?

Field: The modulation derived by Anand, Daniel, and Stephens agrees with that 
assumed for cosmic-ray protons. To change the subject, may I draw attention to the elongated clouds discussed by Verschuur in his report. I am worried about the existence of supersonic velocities, or rather, velocity gradients over very small distances. The observations are done with finite beam widths and therefore one observation includes information on regions quite large distances apart. Consequently, the problem may not be too great, but, on the other hand, the calcium-line profiles indicate a large turbulent velocity in a very small angular region. Is it possible that one can have large amplitude hydromagnetic waves, basically transverse waves, which could be supersonic and not dissipate at the same rate as compression waves? Nevertheless, for a cloud with $10 \mathrm{~cm}^{-3}$ and $B=5 \mu \mathrm{G}$, the Alfvén velocity $V_{A}=3.0 \mathrm{~km} \mathrm{sec}^{-1}$, which is not much more than the thermal speed.

Pikel'ner: According to a paper by Heiles (1967), the average density in clouds is between 2 and $4 \mathrm{~cm}^{-3}$. These values will increase $V_{A}$ by a factor of about 2. (Heiles, C.: 1967, Astrophys. J. Suppl. Ser. 15, 97.)

Weaver: I agree with Pikel'ner on the densities he quotes. Such values seem to be indicated by the observations.

Van Woerden: I think there is still evidence for hydrogen densities of 10 or $20 \mathrm{~cm}^{-3}$ in clouds.

Woltjer: I have a question to Parker. If, as Verschuur has discussed, there is some evidence for a helical field in the neighborhood of the Sun, you could think of a configuration where the field is force-free in the $z$-direction. Have you considered such fields and would they affect your conclusion concerning the strength of the field?

Parker: I have considered such fields, but they do not offer possibilities to increase the magnetic field strength. The point is, if you twist a helix until it really becomes force-free, and external pressures are no longer required to confine it, you will have increased the pressure in the direction along the helix by an equal amount. And there is still an instability which tends to make things bulge, i.e., you have the same problem as before.

Woltjer: Except that in the plane of the Galaxy you have to take into account differential rotation, and a very small change in rotational velocity gives you a comparatively large dynamical effect.

Parker: That is true. But, if you have pressures within the disk of the Galaxy, the gas-field system is unstable through loops bulging out in the $z$-direction without significant change in the instability criterion. And you lose your force-free configuration as soon as it becomes unstable.

Van de Hulst: I have a question to Parker. Parker said in his lecture that sheets of matter might be envisaged which are oriented along the gravity vector and the magnetic field lines. Could you suggest any observational program to check that prediction?

Parker: That is a question that I am interested in pursuing here. The linear analysis of a disk with a uniform field shows that the most unstable modes are vertical sheets. I have from time to time asked various observers whether or not this is something that you could see. I don't know whether the sheets would have enough relative 
velocity to be separated by Doppler shifts or not; and it is not obvious to me how one would go about detecting the sheets. Nor is it obvious that vertical sheets still persist in the ultimate, very non-linear dynamical state just because the linear analysis for small perturbations indicates their existence.

Weaver: In the velocity-latitude diagrams of the kind that $I$ have been showing in my report (see Figure 3 at p. 27), isolated velocity peaks are seen extending over a considerable interval in $b$ (or in $l$ ). This is very suggestive of a sheet, but one may easily be deceived since the nature of the diagram does not give direct information on the hydrogen distribution in depth along the line of sight.

Van de Hulst: Dr. Parker, is the dust also tied to the magnetic field so that we can say that the dust must follow roughly the same spiral pattern? Also, you mentioned the possibility of a magnetic field of $1 \mu \mathrm{G}$. Is that not ruled out by the spectrum of cosmic rays at very high energies, or do you think that at very high energies the cosmic rays are intergalactic?

Parker: I will answer your last question first. The reduction of the magnetic field from, say $4 \mu \mathrm{G}$ to $1 \mu \mathrm{G}$, would have very little effect on the precise value of the energy at which you can no longer contain particles in the Galaxy; roughly speaking the energy goes down by a factor of 3 or 4 . In answer to the first question, the dust is tied to the magnetic field if photoelectric effects give each dust grain a charge of at least one electron. In fact, without a magnetic field one cannot understand striations, and one can put a lower limit of about 1 or $2 \mu \mathrm{G}$ in the case of fine striations in the Pleiades. I imagine that one could put lower limits in a similar way over a good part of the Galaxy.

Van de Hulst: Parker mentioned that the ratio of cosmic-ray pressures, $\left|P_{\|}-P_{\perp}\right| \mid$ $P_{\|}$, is $10^{-2}$ or less. He suggested that isotropizing instabilities bring any anisotropy down to $10^{-2}$ in $10^{2} \mathrm{yr}$ and down to $10^{-3}$ in $10^{6} \mathrm{yr}$. If the observations are good enough to determine an anisotropy of $10^{-3}$ or less, this measurement is very important for the origin of cosmic rays. A few years ago it was claimed that anisotropy of this order had been detected and at succeeding cosmic-ray conferences this claim has been repeated. But can Dr. Parker tell us what the practical limitations are? Is the value unknown just because people have not spent enough effort, or is it too difficult, or what?

Parker: The difficulty in observing the anisotropy of cosmic rays is that we are sheltered in the solar system by a field of $50 \mu \mathrm{G}$ over scales of $10^{13} \mathrm{~cm}$. We are in a shelter with the wind blowing by outside, if you want to look at it that way. You cannot expect to see the anisotropies for particles whose cyclotron radius in a $50 \mu \mathrm{G}$ field is less than $10^{13} \mathrm{~cm}$, and therefore you must observe particles above about $0.5 \times 10^{12} \mathrm{eV}$. The cosmic-ray spectrum drops off steeply with energy; the number of cosmic-ray particles above an energy $E$ declines as $E^{-1.7}$. There are many measurements up to about $0.2 \times 10^{12} \mathrm{eV}$. Unfortunately the interpretation of these measurements in terms of anisotropies is complicated by the effects of the solar magnetic field. But some equipment exists, built for neutrino experiments, which may have a suffciently high counting rate at $10^{12} \mathrm{eV}$ to look for anisotropies of the order of $10^{-3}$ or $10^{-4}$; the equipment has not yet been used for that purpose. At the present time, 
based on measurements at $0.2 \times 10^{12} \mathrm{eV}$ it looks as though the anisotropy is less than or equal to $10^{-3}$. It will probably be a couple of years before there is better information. I quite agree with van de Hulst that from anisotropy information one might learn a great deal about the origin and behavior of cosmic rays in the Galaxy.

Tsytovich: Dr. Parker, have you estimated these isotropization times of $10^{2}$ and $10^{3} \mathrm{yr}$ from the linear approximation to the instability theory?

Parker: My estimate is based on the calculations of Lerche, who used a purely linear theory, not a quasi-linear theory. I would be most interested to hear your comments.

Tsytovich: There is experimental evidence that in some cases a linear theory does not yield a reliable estimate of the relaxation time. For example, let us consider isotropization and relaxation of a beam in a plasma. The plasma particles have a Maxwellian velocity distribution, and the beam provides a second hump in the velocity distribution at the beam velocity. As Kadomtsev has pointed out in his Report (see Section 3a, p. 112), the second hump develops rapidly into a velocity-plateau; the energy of the beam is converted into turbulent and thermal energy and the beam is isotropized. Now if one applies this theory to solar bursts of type III, one finds that the beam extends over a distance three orders of magnitude larger than follows from the linear approximation. Therefore non-linear stabilization exists. It can be shown that non-linear stabilization occurs for those beams for which $V>V_{\text {crit }} \approx 3\left(m_{i} / m_{e}\right)^{1 / 4} V_{T e}$, where $V_{T e}$ is the thermal velocity of the electrons; $V_{\text {crit }}$ is the velocity at which non-linear coupling between electrons and ions occurs. Observations of solar bursts of type III confirm this predicted behavior. If the temperature is not too high $V_{\text {crit }}<c$. Since the velocity of cosmic rays is close to that of light, non-linear stabilization occurs for cosmicray instabilities. Therefore the isotropization of cosmic rays may take a much longer time, up to 3 orders of magnitude, than predicted from the linear theory.

Pikel'ner: May I bring up a question about the Rayleigh-Taylor instability? When this instability develops, there are two possibilities. First, compression of the magnetic field may push out gas and field at the other side of the galactic plane. Therefore, the magnetic field will not be increased considerably in massive clouds. But consider a second case in which the gravitational forces, associated with the gas, are so strong that they 'attract' the perturbation at the other side of the galactic plane. Then we have compressed gas together with a compressed magnetic field. To find out which of these possibilities is realized we probably have to rely on observations.

Mestel: I would like to enlarge on Pikel'ner's remark about self-gravitation. Consider a region with a large-scale galactic magnetic field $\mathbf{H}_{\boldsymbol{i}}$ and an associated density $\varrho_{i}$. Now let the Field-Pikel'ner instability start locally, so that a massive blob begins to contract, causing a local distortion in the magnetic-field structure. For simplicity we assume the blob is spherical and cool enough for thermal pressure to be ignored. From the virial theorem we have a simple criterion relating the mass $M$ and the magnetic flux $F$ in order that indefinite gravitational collapse can ensue:

$$
{ }_{5}^{9 \pi^{2}} G M^{2}>F^{2}
$$


This may be written alternatively as

$$
\eta \equiv \frac{8 \pi^{2}}{3} \frac{G R^{2} \varrho_{c}^{2}}{H_{c}^{2}}>1,
$$

where $R$ is the radius of the sperical blob, $H_{c}$ the central field-strength and $\left(\varrho_{c}+\varrho_{i}\right)$ the central density (see Figure 1). Thus with $\eta>1$, indefinite collapse can occur, and the

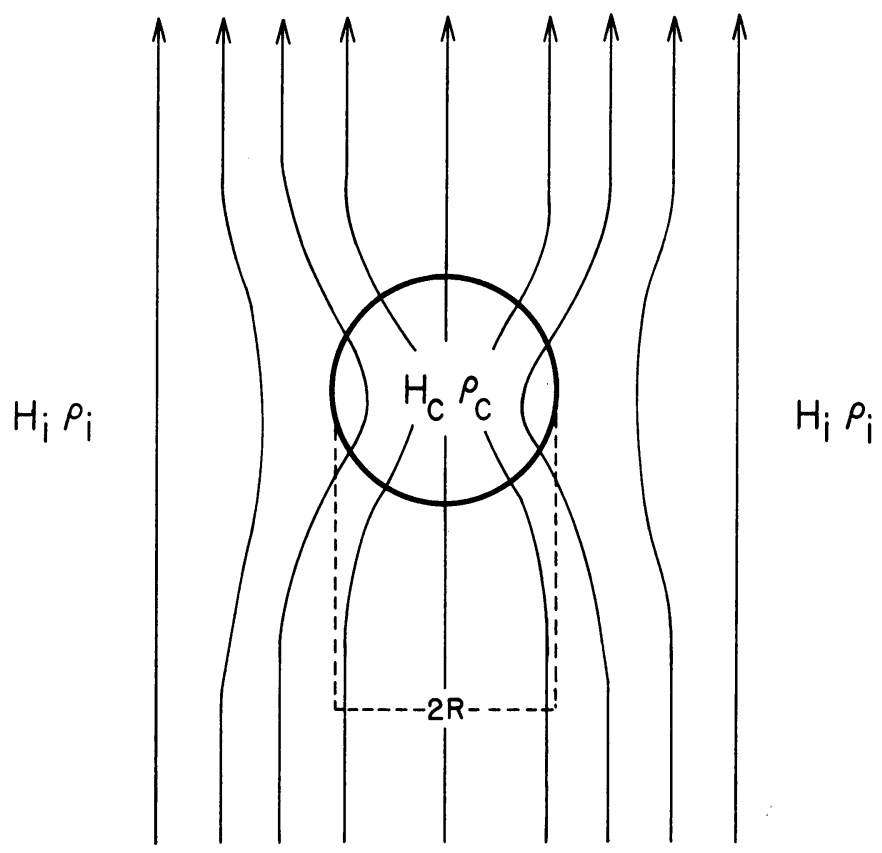

Fig. 1. (See the remark by Mestel.) Magnetic-field strength in a contracting cloud.

cloud can ultimately break up into a proto-star cluster (by flow down the field, if flux-freezing continues to hold). But if $\eta<1$, the collapse must be halted when the distorted field exerts forces strong enough to balance its self-gravitation. This magneto-gravitational equilibrium is achieved when

$$
\frac{\varrho_{c}+\varrho_{i}}{\varrho_{i}}=\frac{1}{1-\eta^{1 / 3}}
$$

and

$$
\frac{H_{c}}{H_{i}}=\left(\frac{1}{1-\eta^{1 / 3}}\right)^{2 / 3}
$$

I suggest that this model describes approximately what Verschuur has discussed in his Report (p. 157). An increase of 5 in the field-strength requires a density increase (in this spherically-symmetric model) of $(5)^{3 / 2} \approx 11.25$. The mass required to maintain this local field distortion is $\approx 0.87$ of the initial value for indefinite collapse. Verschuur 
drew my attention to the paper by Clark (1965) that discussed the parameters of the first two clouds in which later the Zeeman effect was found. It is gratifying to see that one cloud has an estimated mass of $2500 M_{\odot}$. The other one has only $800 M_{\odot}$, and this mass is too small to maintain a distorted field. However, Schmidt pointed out to me that since Clark has only lower limits on his cloud diameters, the masses are correspondingly lower limits also. I would like to see some detailed models computed of such clouds, including thermal pressure, especially to clarify the conditions under which the clouds are oblate or prolate about the field direction. (Clark, B. G.: 1965, Astrophys. J. 142, 1398.)

Field: In the equation for the critical mass, presented by Mestel [his Equation (2)], the term $R \varrho_{c}$ represents mass per unit area. This parameter is observed directly by the 21-cm observers. One can therefore re-examine Verschuur's Table III and see whether Mestel's criterion applies. In a preliminary list of 14 clouds measured by Verschuur, roughly half had large values of the magnetic field. In every case $R \varrho_{c}$ exceeds the value corresponding to $3 \mu \mathrm{G}$ in Mestel's equation. On the other hand, when the mass per unit area is small, the observed magnetic field is about $3 \mu \mathrm{G}$. I therefore support Mestel's suggestion that in the clouds of low surface-density one is observing essentially the intercloud magnetic field; in the cases of high surface-density we witness contraction of the magnetic field. I have also considered a little bit the problem of expanding Mestel's theory by inclusion of the gas pressure. The effect of gas pressure will be to expand the gas along the field line. I find that there is a critical radius of about $4 \mathrm{pc}$ (given the conditions of thermal balance based on cosmic-ray heating). If across the magnetic field a cloud has a radius less than $4 \mathrm{pc}$, gravitational forces are less than the pressure forces along the field. Such a cloud cannot contract, and it will be stretched along the magnetic field into a prolate shape. If, on the other hand, the radius across the field is bigger than $4 \mathrm{pc}$, the radius along the field depends on the surface density but it has a maximum of $4 \mathrm{pc}$, which is attained when the surface density, is becoming so large that contraction across the field can occur. It follows that clouds extending more than $4 \mathrm{pc}$ across the field, and having surface density sufficient for gravitational contraction to occur, will be oblate, with the minor axis along the field. Hence observers may want to study the shapes of the clouds which have large magnetic fields.

\section{The Galactic Halo}

Mestel: During the third Symposium in this series, in 1957, there was a.long debate between Spitzer and Pikel'ner on the nature of the galactic halo. I would like to ask the observers: what has happened to the idea of a $10^{6} \mathrm{~K}$ halo? One of the reasons for introducing it was, I think, to maintain the disk of neutral hydrogen at a constant thickness over the galactic radius, in spite of the variation of the $z$-component of the gravitational field. Do the new 21-cm observations of spiral arm thicknesses destroy this argument?

Verschuur: There is no halo!

Mills: There is a halo! 
Burke: Perhaps I can try to summarize the discussion on the interpretation of our data, that Mills and I have had running over several years. The data of Mills supported the idea of a halo, but came only from the Southern Hemisphere observatories. Turner and I made a 200-MHz survey of the sky from the Northern Hemisphere (Burke, 1967) which showed no evidence for the original concept of a halo, that is, an object more or less spheroidal in shape with a radius of 16 or $20 \mathrm{kpc}$. However, a flattened, spheroidal distribution some one or two kpc thick, is allowed by the data. Perhaps Dr. Mills would like to comment? (Burke, B. F.: 1967, in Radio Astronomy and the Galactic System, IAU Symposium No. 31 (ed. by H. van Woerden), Academic Press, London, p. 361.)

Mills: Yes. Since the earlier work of about ten years ago, only one set of systematic southern observations has been made. They have just been completed by Hamilton using the Parks 210 -foot reflector at various frequencies (principally $150 \mathrm{MHz}$ and lower). Hamilton's paper is at present in press and I have no detailed information about it. But it does seem that the isophotes at the southern galactic latitudes around the galactic center show evidence for a rather flattened, small halo or corona. I certainly agree with Burke that there is no evidence for a very large halo. If it exists, it is fairly small. In the disk it has a radius to the point of half emissivity of the order of $10 \mathrm{kpc}$; perpendicular to the disk this radius is about $5 \mathrm{kpc}$. The axial ratio is thus about $2: 1$.

Menon: If the concept of a halo, especially that of an extensive halo, is rejected, then the original problem of Spitzer (1956) is unsolved, namely that of keeping the gas down in a layer of constant thickness. In addition we know now that the cosmic rays also exert pressure, and Spitzer's problem becomes even more important. (Spitzer, L.: 1956, Astrophys. J. 124, 20.)

Field: I have done some calculations on the back of an envelope, which indicate that a halo of $10^{6} \mathrm{~K}$ and a number density of $10^{-3} \mathrm{~cm}^{-3}$ is detectable by current $\mathrm{X}$-ray techniques. Such experiments should be done. In addition to the (historical) argument mentioned by Menon, I want to add another of Spitzer's arguments, that of the confinement of clouds at high latitudes. There are clouds known at $z$-distances larger than $1 \mathrm{kpc}$ (Münch and Zirin, 1961) and the original Spitzer proposal was to confine these clouds by outside pressure produced by the hot, rarefied halo gas.* (Münch, G. and Zirin, H.: 1961, Astrophys. J. 133, 163.)

Syrovat-skii: For a long time one of the arguments in favor of a halo has been that one needs a place to store all cosmic rays. I want to point out that this argument is no longer valid as can be shown from age determinations of cosmic rays. At the recent Budapest Conference on Cosmic Rays, Shapiro and others reported that the age of cosmic rays is less than $50 \times 10^{6} \mathrm{yr}$; the age may be $1 \times 10^{6} \mathrm{yr}$, perhaps $3 \times 10^{6} \mathrm{yr}$. These results were based on measurements of the abundance ratios $\mathrm{Li} / \mathrm{B}$ and $\mathrm{Be} / \mathrm{B}$ in the cosmic-ray flux. A time of $1 \times 10^{6} \mathrm{yr}$ or $3 \times 10^{6} \mathrm{yr}$ is needed by the cosmic rays

* 21-cm observations of three of these clouds (Habing, H. J.: 1969, Bull. Astron. Inst. Netherl. 20, 177) show internal velocities so large that the clouds cannot be confined by a halo of the proposed properties. (Ed.) 
to get out of the galactic disk. Therefore it seems unlikely that the cosmic rays spend a long time in the halo.

In addition I should like to point out that the existence or non-existence of the halo has no influence on the estimate for the energy output $U$ (dimension: $\operatorname{erg~sec}^{-1}$ ) of cosmic-ray sources in the Galaxy. If the energy density of the cosmic rays $W_{c r}$ (for which we take the local value, $1 \mathrm{eV} \mathrm{cm}^{-3}$ ), and if the age of the cosmic rays is $t \mathrm{yr}$, then $U=W_{c r} V t^{-1}$, where $V$ is the volume of the Galaxy in which the rays are found. But this expression is equal to $W_{c r} M_{g} c x^{-1}$, where $M_{g}$ is the total amount of interstellar gas in the Galaxy, $c$ the velocity of light, and $x$ the amount of matter, traversed by the cosmic rays during their existence ( $x=c \varrho_{g} t$, where $\varrho_{g}$ is the mass density of the interstellar gas). From the abundances in the cosmic-ray gas it follows that $x$ is between 3 and $10 \mathrm{~g} \mathrm{~cm}^{-2}$ and if we take $M_{g} \approx 10^{10} M_{\odot}$ then we find $U=3 \times 10^{40} \mathrm{erg} \mathrm{sec}^{-1}$. This estimate is independent from the existence or non-existence of the halo.

Weymann: In this connection may I ask Dr. Parker the question whether or not the escape of cosmic rays from the disk of the Galaxy leads to an extensive halo?

Parker: I cannot tell you the extent of the halo produced by the cosmic rays, because I do not know how to compute the extent of the cosmic-ray inflated bubbles at the moment when they are cut loose from the Galaxy by a plasma instability. I suggest that the inflated field will extend up at least a few hundred pc.

Woltjer: How does the bubble cut itself from the plane? Don't you run the risk that, once you have the bubble, you have a permanent hole?

Parker: The topology of the magnetic field, I think, prevents any permanent hole, at least with any scheme that I have been able to think of. Perhaps you have something in mind?

Davis: After the cosmic rays have escaped, does one not arrive at a configuration like that in the Sun after the solar wind has blown out a weak field loop? You get streaming lines of force that go out forever and probably have enough wiggles in them so that the cosmic-ray gas cannot flow out very fast.

Verschuur: Dr. Parker, how could one observe those bubbles?

Parker: They might emit some synchrotron radiation, but I am not sure. Presumably the thermal gas has slid down the lines of force, and there is not much thermal gas remaining in the bubbles. Let me think first about the escape of cosmic rays in a weak field $(B \lesssim 1 \mu \mathrm{G})$. In this case the cosmic rays are confined more by friction with the thermal gas than by magnetic field pressure. The friction might possibly lift some gas up into the halo; and then (coming back to a remark that Field made) you might see gas raining down upon you in some condensed state, as you see in the solar corona. One could imagine processes like that, even if the field were several $\mu \mathrm{G}$. There is an infinite number of alternatives at this point, and I think we should be very cautious in pursuing any one of them.

Field: Would one not expect filaments of neutral gas raining down on the galactic plane along the magnetic field? And is there not evidence consistent with this?

Verschuur: I cannot picture a bubble configuration that accounts for the data. Some relevant questions are: given the distribution of intermediate- and high- 
velocity clouds, what is the three-dimensional picture of the magnetic field and where are we situated? Besides these questions, there is the problem that one might not get sufficiently large velocities during the streaming downwards.

Weaver: Certainly the idea of gas flowing into the local gas structure is completely consistent with the observations. But the observed velocities are very high, often up to 50 and $60 \mathrm{~km} \mathrm{sec}^{-1}$, not infrequently $100 \mathrm{~km} \mathrm{sec}^{-1}$.

Field: I think you are quite right about high-velocity or intermediate-velocity clouds. But is there not a general inflow at about 7 to $10 \mathrm{~km} \mathrm{sec}^{-1}$, both above and below the plane, observed by Dieter and, I believe, the southern observers?

Weaver: I do not know about a specific inflow velocity. The point is that there is always a negative velocity tail in the velocity distribution, and perhaps this tail represents the falling down into the galactic plane.

Van de Hulst: If the matter rains down somehow in the form of filaments, then you can see one of those filaments in projection and see an elongated cloud. If, on the other hand, it moves down in the form of a plane sheet, containing the direction of gravity and the direction of the magnetic field, then we may observe this sheet from two directions. Looking along the galactic plane perpendicular to the field, you don't see the sheet elongated. Looking along the galactic plane and along the magnetic field you see it elongated, but then there is not a projected magnetic field component. And so, in either case, you cannot see what Verschuur mentioned in his Report. Therefore it is not clear to me what the actual interpretation is of the elongated clouds which Verschuur has demonstrated.

\section{Origin of the Magnetic Field}

Woltjer: I have a question referring to the primordial field. It sounds very reasonable that the present field is in the azimuthal direction, just because of galactic differential rotation. But during the history of the Galaxy an originally uniform field will have undergone a large number of windings and, therefore, if you look in the neighborhood of the Sun, between 10.0 and $10.1 \mathrm{kpc}$, you would have the field in one direction; between 10.1 and 10.2, in the opposite direction and so on. This would create very conspicuous effects in the Faraday rotation pattern, which are not in fact observed. Does Dr. Verschuur agree?

Verschuur: You are correct, in the plane there are only a few radio sources, which are not polarized.

Syrovat-skii: Parker mentioned three possible mechanisms for the origin of the magnetic field. I want to add a fourth - supernova explosions. During the lifetime of the Galaxy there have been $3 \times 10^{8}$ supernova explosions, if the frequency of this event is one per $30 \mathrm{yr}$. Let us take $10^{67} \mathrm{~cm}^{3}$ for the total volume of the Galaxy. Then we have one supernova event per $3 \times 10^{58} \mathrm{~cm}^{3}$, which is the volume of a sphere with a radius of $6 \mathrm{pc}$. The Crab nebula (assuming it can serve as a prototype) has, at present, a volume with a diameter of $1 \mathrm{pc}$ and a magnetic field of $300 \mu \mathrm{G}$. If it expands to a diameter of $10 \mathrm{pc}$ the field will be $3 \mu \mathrm{G}$, a value equal to that of the interstellar field. 
So it seems possible to obtain the average flux density of the magnetic field of the Galaxy from independent supernova explosions. But there is the observation of the regularity of the magnetic field. Why is the field regular if individual supernovae produce it? Perhaps there is some correlation between the orientations of stellar angular momenta, reflecting stellar formation in the differentially rotating Galaxy. Then we can expect correlation between partial magnetic fields and the existence of some mean field.

Pikel'ner: But if there is such a correlation, then you would expect the total efficiency of this process to be much lower than you assumed.

Syrovat-skii: Yes, but only for the mean field.

Kardashev: I should like to draw attention to the nucleus of the Galaxy. It probably has a regular field which, near the nucleus, may be dipolar. At large distances from the nucleus the field will be twisted. Perhaps it extends out as far as the $3 \mathrm{kpc}$ arm. The field may be similar to the magnetic field generated in a supernova explosion, as described in papers by Piddington (1966) and by myself (Kardashev, 1964). The main process is twisting of the magnetic flux that connects the fast-rotating stellar remnant with the envelope. Near the remnant we have a dipole field and $B \propto R^{-3}$, where $R$ is the distance to the remnant. Farther out there is a radial component $B_{r} \propto R^{-2}$, and a tangential component $B_{t} \propto R^{-1} . B_{t}$ increases linearly with the number of revolutions made by the stellar remnant. [Kardashev, N.S.: 1964, Astron. Zh. 41, 807 (1965, Soviet Astron. 8, 643); Piddington, J. H.: 1966, Monthly Notices Roy. Astron. Soc. 133, 163.]

Ozernoi: First, if the magnetic fields arise in supernova explosions, then we need very large magnetic fields for the pre-supernova stars. Then we are left with the question where this embryonal field comes from. Second, if galactic magnetic fields arise from explosions in the nucleus, then irregular galaxies without nuclei cannot have magnetic fields. But they do have magnetic fields, because, for instance, they have large amounts of non-fragmented gas.

Zel'dovich: Dr. Ozernoi, indirect evidence is not enough in such an important problem! Let me present my opinion. There is a problem with the time scale for the production of the magnetic field in the Galaxy. Together with Sagdeev and Pikel'ner I made some calculations on the turbulent dynamo proposed by Steenbeck et al. (1966). The characteristic rotation time of the Galaxy is about $2 \times 10^{8} \mathrm{yr}$ and the characteristic turbulence time is $10^{7} \mathrm{yr}$. Since the efficiency of the dynamo is low (less than 0.1) there is little amplification. But in the nucleus of a galaxy the time scales become more favorable. So I suggest that in the nucleus of a galaxy a primordial field is created (e.g., by thermo-electric effects, or by rotational friction of electrons against the $3 \mathrm{~K}$ radiation). This field is amplified by nuclear rotation and flows out of the nucleus, together with matter. This suggestion would imply that galaxies without a nucleus have no magnetic field. (Steenbeck, M., Krause, F., and Radler, K. H.: 1966, Z. Naturforsch. 21, 369.)

Verschuur: I think that observations by Mathewson (unpublished) indicate polarization of starlight in the Magellanic Clouds. If I am not mistaken, he finds a corre- 
lation of polarization with spiral arms in the Large Cloud. And the Large Magellanic Cloud has no nucleus. (Polarization measurements of stars in the Magellanic Clouds have been published by Th. Schmidt: 1970, Astron. Astrophys. 6, 294.)

Menon: In addition, the Magellanic Clouds, as well as other irregular galaxies, are non-thermal radio sources. So they contain magnetic fields.

Grebinskii: I should like to point out that dynamos are unlikely to work on the galactic scale, because the galactic rotation is so slow. For example, Brajinsky's investigation of a terrestrial dynamo shows that it takes an extremely long time - of the order of 1000 revolutions - to reach a stationary state.

Tsytovich: There is the possibility of generating magnetic fields by plasma turbulence. The time scales are very short. The generated fields can be regular if the original turbulence field was regular. If somewhere there is turbulence (e.g., Langmuir turbulence; see the Introductory Report by Kadomtsev and Tsytovich, p. 108) the currents in the plasma produce certain forces, which react on the electrons and therefore amplify the currents. This generates a magnetic field. Then, an additional possibility exists: turbulent plasma may become unstable and separate into (a) regions with higher plasma densities and low turbulent-energy densities and (b) with lower plasma densities and higher turbulent-energy densities. The same thing can happen with respect to regions of different magnetic energy densities. The growth rate of the instability is proportional to $k \approx 1 / L$, where $L$ is a dimension of the created magnetic field. Therefore smaller scales grow faster.

Pikel'ner: But Dr. Tsytovich, this produced field will be of small scale and so the problem is: how do we get a general field?

Tsytovich: I do not know. I only want to point out that in a turbulent plasma magnetic fields can be produced in a short time. But I admit, they have small dimensions.

Woltjer: In the amplification of the magnetic field there are two time scales. You can increase the magnetic energy of the field on the kinematical time scale, $L / v$. You can increase the magnetic flux only on the time scale on which effects of finite conductivity become important. Unless you can make this time scale short, I cannot see how you can increase the flux of the galactic magnetic field appreciably, and, therefore, I cannot quite see how all these models and explosions from galactic nuclei and similar effects can give you a magnetic field of any sizeable scale.

Zel'dovich: I am not sure that you are right. Of course we know that axisymmetric dynamos do not work, and neither do plane dynamos. But there exist three-dimensional motions, which are able to act as a dynamo. And in addition, we always speak about collisional conductivity, but might not turbulence increase the resistivity and decrease the conductivity and so decrease the time scale for creation of magnetic flux?

Woltjer: In answer to Zel'dovich: the three-dimensional dynamo will not work if you have infinite conductivity. It is a dynamo obtained by balancing terms that involve conductivity.

Parker: Woltjer is right, except that the growth time of the dynamo refers to the 
smallest participating turbulent element. So in effect there is no limitation on the time.

Woltjer: But you get very small scales.

Parker: I think that reasonable growth times of less than $10^{8} \mathrm{yr}$ can be obtained for scales of $1 \mathrm{pc}$, if one puts in ambipolar diffusion and everything else.

Csada: I want to discuss a three-dimensional, periodic solution of the magnetohydrodynamic equation of motion, which solution may act as a model for the dynamo of the interstellar magnetic field. The method of solution is a continuation of the 'eigenvalue' theory of Elsasser (1946) and Bullard (1955). Jayanthan's work (1968) differs basically from mine. Part of my results have been published previously (Csada, 1966).

The mathematical problem is to solve the equation

$$
\frac{\partial \mathbf{H}}{\partial t}-\operatorname{curl}(\mathbf{v} \times \mathbf{H})=\kappa \Delta \mathbf{H}
$$

by the series

$$
\mathbf{H}=\sum_{n=1}^{\infty} B_{n}(t) \mathbf{H}_{n}(x, y, z) .
$$

The velocity $\mathbf{v}$ is a known function of the coordinates, $\kappa$ is the resistivity $\left(\kappa=c^{2} / 4 \pi \sigma\right)$ and $\mathbf{H}_{n}$ is a certain given set of orthogonal functions. I have been able to show that a velocity model $\mathbf{v}(x, y, z)$ can be found such that the functions $\mathbf{B}_{n}(t)$ are periodic and stable, provided that the conductivity is infinite. For finite conductivity, dissipation of magnetic energy occurs. In this way a dynamo is obtained, in which the velocity field and the magnetic field are consistent with Equation (1). For a detailed analysis, suitable for discussion of actual observations of interstellar matter, a much more complicated field pattern may be required. [This remark has been condensed (Ed.).] (Bullard, E. C.: 1955, Proc. Roy. Soc. London Ser. A233, 285; Csada, I. K.: 1966, Bull. Astron. Inst. Csl. 17, 321 ; Elsasser, W. M.: 1946, Phys. Rev. 69, 106; Jayanthan, R.: 1968, Monthly Notices Roy. Astron. Soc. 138, 477.)

Burke: So far everybody seems to agree that there is a general magnetic field. But an important aspect of Verschuur's report was that there is very good evidence for a magnetic field with a scale of the order of a few hundred parsecs, but not for a field on a considerably larger scale.

Verschuur: Let me extend Burke's argument. Why are magnetic fields permanent? Can't they be transient? We now believe that the once permanent $\mathrm{HI}$-clouds are also transient.

Parker: Once you have magnetic fields, it is hard to get rid of them, in fact equally as hard as to create them.

Greenberg: In M31, the Andromeda galaxy, optical polarization follows the spiral arms. This shows the existence of large-scale magnetic fields.

Woltjer: Optical polarization does not allow you to determine what the magnetic flux is. You have exactly the same polarization, whether all your field-lines are parallel or whether half of them are opposite to the other half.

Pikel'ner: I do not like the idea of an ensemble of independent local fields. I am 
afraid that it would be difficult to explain the diffusion of the cosmic rays and the uniformity of the spectra of relativistic electrons. In addition cosmic rays will leave the Galaxy in the neutral surfaces between the local fields.

I would prefer the hypothesis of the primordial origin for the magnetic field. As an argument in favor of this hypothesis I would propose the existence of bridges between galaxies. These bridges cannot be understood without a magnetic field between galaxies, and a quasi-regular field of that order cannot have been produced by a dynamo mechanism or any other mechanism. There is one more point: If pulsars are indeed neutron stars with a very strong magnetic field, then this proves that the main part of the magnetic field of the star was in the nucleus and was condensed when collapse took place. Quasi-regular magnetic fields which go through the nucleus of the star argue for the assumption that a field already existed in the gas clouds out of which the presupernova star was formed.

Burke: First, with respect to the extra leakage of cosmic rays through the neutral sheets, I would like to answer Pikel'ner that, if the neutral sheets are separated by something of the order of the width of the arm, then you are increasing the surface through which cosmic rays can escape by a geometrical factor of the order of 2 or 4 . There will not be a serious effect on the required production rate of cosmic rays. The second point concerns the intergalactic bridges, which are not necessarily evidence of magnetic fields. They could be caused by gravitation as well. In Prendergast's numerical experiments (unpublished) short-living bridge-like structures occur; intergalactic bridges are short living, too, since not all pairs of galaxies show bridges.

Zel'dovich: Is there polarized light in bridges? Are they made out of stars?

Pikel'ner: The bridges are somewhat similar to spiral arms, in that they contain young, hot stars and HiI-regions. Without a magnetic field the gas would turn into stars in a short time.

Zasov: There are some arguments in favor of magnetic connections between galaxies. First, Arp has observed polarization in one bridge. Second, to keep gas inside a thin tube for a long time you need a strong magnetic field.

Van Woerden: How do you know the gas is kept together for a long time?

Zasov: The frequency of bridges is very high. Several percent of the galaxies show bridges or tails.

Burke: As far as I remember intergalactic bridges are observed between close pairs of galaxies. A. Toomre (unpublished) has shown that a close encounter between the Magellanic Clouds and our Galaxy can draw a string of material out of our Galaxy. This process requires only gravitational forces.

\section{Summary}

[On September 16 a group gathered to discuss informally galactic magnetic fields. On September 17 Dr. E. N. Parker presented a summary to the formal meeting. His summary and the ensuing discussion appear here in the Proceedings instead of in their chronologically correct place (Ed.).] 
Parker: Several participants interested in galactic magnetic fields met to discuss this subject. We hoped to express and to clarify our views, to argue, and perhaps to arrive at a consensus. The first question that came up was the old one of whether or not the Galaxy really has a general field; the consensus was that it has. The evidence has been accumulating rather steadily in the past and now it accumulates fairly rapidly. The polarization of starlight and the Faraday rotation measurements would certainly be extremely difficult to understand if there were not a large-scale, i.e., a general field in the Galaxy; I might add that cosmic rays would also be difficult to understand. Their persistence in the solar system for so many (up to $10^{9}$ ) years, would also be difficult to understand without making rather grandiose assumptions. I think, therefore, that everyone would agree that there is apparently a general field in the Galaxy. When one comes to the more detailed question of the structure of that field, however, some points are debatable. But there seems to be agreement that the simplest picture one can form for the galactic field, or for the observable fields in the neighboring arms of the Galaxy, is that, to a first approximation, there is a longitudinal field along the direction of the arms. The field may not always have the same sense but the lines of force seem to lie along the arm. As Weaver mentioned (this volume, p. 22), the Sun seems to reside in a local spur. Some have suggested that in this Spur the field is a tightly wound and somewhat stressed helix or, perhaps, a uniform field through that spur with a local loop. We made no attempt to hammer out which of these pictures fits most closely. At the present time, we seem to be in a spur which has a field of a somewhat more complicated form, perhaps, than the neighboring arms. But, on the other hand, one will have to see what future observations show in the details of the fields in the local arms. An important point, when we begin to think about the significance of the magnetic field, is that the energy density of the magnetic fields, based on the observational indications of a few $\mu \mathrm{G}$ is comparable to the turbulent energy density of the interstellar medium. That is, if you multiply cloud velocities times mean, smeared-out densities, you get $10^{-12} \mathrm{erg} \mathrm{cm}^{-3}$, as van de Hulst emphasized in his Report (p. 3). And these two energies, the magnetic energy and the turbulent energy, are comparable to the cosmic-ray energy. The thickness of the disk of the Galaxy seems to be determined by the sum of these energies, plus the cosmic-ray energy density. All three inflate the disk of the Galaxy. Very roughly, there is an upper limit on the strength of the field of 5 or $6 \mu \mathrm{G}$, if we are to understand the observed thickness of the Galaxy in terms of the observed densities of material, etc. That number may change somewhat when observational values change, but it is the right order of magnitude.

Then we spent considerable time discussing a lower limit on the field, i.e., the question of how weak the field could be and still perform the duties that we think it performs. There are a number of arguments that one can give. In our discussion, I took a point of view that I do not normally hold. I argued that the field in the Galaxy could be very weak, with a field strength of only $1 \mu \mathrm{G}$. It is clear, however, that with such a weak field present it is difficult to understand the more or less steady, stagnant nature of the cosmic ray gas; although by appealing to plasma turbulence, one might be able to do so. Other members of the group added some very sound arguments 
which probably make the weak, $1 \mu \mathrm{G}$ field an untenable hypothesis. Among the points made was that you would have difficulty explaining the non-thermal radio emission, if the field were weaker than about $3 \mu \mathrm{G}$. Another point was that if the field were only $1 \mu \mathrm{G}$, it would be completely at the mercy of the turbulence in the interstellar medium. It would be much too weak to resist the stresses; and the turbulence would therefore very quickly tangle up the field, so that there would be no recognizable pattern. We know, however, that in fact there is some tendency for the field to lie along the arms, and that there are sharp reversals of fields. Reversals in the sense of the direction of the field would be quickly obliterated by the turbulence in the interstellar medium. Roughly speaking, the field must therefore be $3 \mu \mathrm{G}$ or more, if it is to maintain its integrity in the face of the turbulence. Unless I am mistaken, then, the consensus of the group was that, for a variety of reasons, the field lies between 3 and 5 or $6 \mu \mathrm{G}$, and that, if it should lie outside that range, we must make complicated assumptions about what we see.

Van Woerden: Where does this field of 3 to $6 \mu \mathrm{G}$ apply? Apparently not in the densest clouds as Verschuur (p. 150) stated, nor in the interarm regions. Should it apply anywhere within the Orion branch, or possibly within a normal spiral arm?

Verschuur: Yes, it should.

Van Woerden: If it is correct that the field between the arms is much smaller than in the arms, the non-thermal radiation between the arms should also be much smaller, a conclusion which fits rather well with the older ideas of Mills.

Pikel'ner: The field between the arms should be less than in the arms. It can be shown by the equilibrium conditions that the density of the gas between the arms is low and no equilibrium is possible for strong fields, especially if the density of cosmic rays is more or less uniform in the galactic disk.

Field: It is still not clear to me whether or not we have information about the field in other spiral arms, other than that it is preferentially along the galactic plane. It seems to me that the information from the Faraday rotation pertains almost entirely to the nearest $500 \mathrm{pc}$ or so and does not extend to other spiral arms.

Verschuur: The Zeeman data which I have on other spiral arms (and there are about four or five clouds which I know must be in other spiral arms) are not inconsistent with a longitudinal field running in a clockwise direction as viewed from the galactic north pole.

Parker: To what distances does the polarization of starlight establish a direction of the field? As I recall, the stars that were observed ranged up to at least one kpc. That observation dictates that the mean distance which you are seeing is of the order of 500 pc.

Verschuur: The stars observed extend up to 3 or $4 \mathrm{kpc}$, but the helical model applies to within $500 \mathrm{pc}$. And it appears, as I tried to point out in my lecture, that stars beyond that may be predominantly influenced by the local field and its polarizing material.

Mestel: Does the field run along the spiral arm, or does it actually run in the toroidal direction? Remember that the angle between the Lin wave and the toroidal direction is small. Non-uniform rotation acting steadily on a field with a toroidal 
component will make the field point in a nearly toroidal direction; whereas the inclination of the gravitational spiral arm is presumably determined by the large-scale structure of the stellar background. There is no obvious reason why the direction of the field and of the arm should coincide; perhaps in a spiral galaxy with less tightly wound arms, the two directions would be markedly different.

Verschuur: If you indeed have a magnetic field which is inclined relative to the gravitational spiral arms and you inject relativistic electrons, they are going to follow the field lines. The non-thermal emission would not be concentrated in the arms; it would be spread throughout the disk.

Parker: As Pikel'ner has pointed out the field in the interarm region can hardly be as strong as in the arm region. The electrons would not radiate when they were in the interarm region; they would radiate only when they were inside the arm.

Burke: I have a question for someone more skilled in cosmic rays than I. The Sun is not going to stay in a spiral arm for very long; it must wander from spiral arm to interarm space and back to spiral arm. If there should be a weaker magnetic field in the interarm region, there should be fewer cosmic rays. One should, therefore, over a period of $(50$ to 100$) \times 10^{6} \mathrm{yr}$ actually see changes in the cosmic-ray intensity. Is such a change observable?

Parker: The following data are available: you look for radioactive nuclei in meteorites, which can be there only because they were produced by cosmic rays. You pick various nuclei with various half-lives, and you find that in every case the number of those radioactive nuclei is what you expected if that meteorite had been exposed to the present cosmic intensity for a period in excess of the half-life of that specific isotope. This finding, of course, gives you only an average of the cosmic-ray intensity over the half-time considered. Now you ask, what happened over the last $10^{8} \mathrm{yr}$ ? The trouble in answering is that we know the average intensity over the last $10^{7} \mathrm{yr}$ (from some isotope which I cannot recall at this moment) and an average over the last $10^{9} \mathrm{yr}$ (from an Argon isotope). The $10^{7} \mathrm{yr}$ isotope does not go back far enough, and the $10^{9} \mathrm{yr}$ isotope averages over all the $10^{8} \mathrm{yr}$ ripples. Therefore to answer your question we need an isotope with a half-life of $10^{8} \mathrm{yr}$.

Field: Dr. Burke, would you tell us again why you would expect the cosmic-ray flux to vary as the spiral arm went by?

Burke: I was merely following up the consequences of assuming that the magnetic field between the arms is much lower than in the arms. I wanted to examine this question because Pikel'ner has struck on a really very crucial point. If there is magnetic field running through the interarm regions and into the spiral arms, then Robert's picture of a shock wave along the arm may be open to serious questioning.

Pikel'ner: If the magnetic field between the arms is less than within the arms, then the cosmic ray pressure may or may not be less there, because it is dependent on the isotropy of the cosmic rays. If the cosmic rays between the arms are isotropic, they should have the same density in interarm space as in the arms, if, as is more likely, the magnetic lines connect both regions.

The second point I wish to discuss is the importance of the magnetic field for the 
shock wave in front of the arms. According to the Lin theory the jump in velocity is about $10 \mathrm{~km} \mathrm{sec}^{-1}$. Does such a jump lead to a shock in the rarefied interarm gas? And can this shock be responsible for very massive clouds at the inner edge of the arms? If between the arms $B \approx 2 \mu \mathrm{G}, n_{\mathrm{H}} \approx 0.05 \mathrm{~cm}^{-3}$, and $T \approx 10000 \mathrm{~K}$, then the magnetosound velocity is about $20 \mathrm{~km} \mathrm{sec}^{-1}$, and the jump in velocity is not enough to produce a shock wave. As for the second question, let us assume that the shock exists. Then it is difficult to see how we could have the frequently discussed intercloud gas $\left(n=0.1 \mathrm{~cm}^{-3}, \mathrm{~T}=7000 \mathrm{~K}\right)$ behind the shock and very low density interarm gas ahead of the shock $\left(n=0.05 \mathrm{~cm}^{-3}\right)$. For the interarm gas moves into the shock with a speed of $10 \mathrm{~km} \mathrm{sec}^{-1}$ and the shock relation $p+\varrho V^{2}=$ constant cannot be fulfilled, since $p_{2}>\left(\varrho V^{2}\right)_{1}$. If the gas behind the shock is not the intercloud medium then it is difficult to see how massive clouds can be expected at the inner edge of the spiral arm. 